\title{
Structural covariance model reveals dynamic reconfiguration of triple networks in autism spectrum disorder
}

\author{
Zhiliang Long, Xujun Duan, Heng Chen, Youxue Zhang and Huafu Chen*
}

*Correspondence:
chenhf@uestc.edu.cn
Key Laboratory
for Neuroinformation
of Ministry of Education,
Center for Information
in BioMedicine, School of Life
Science and Technology,
University of Electronic
Science and Technology
of China, Chengdu 610054,
China

*Correspondence: chenhf@uestc.edu.c Center for Information in BioMedicine, School of Life Science and Technology, University of Electronic of China, Chengdu 610054,

\begin{abstract}
The data open sharing provides us opportunity to investigate mechanisms underlying autism spectrum disorder (ASD) by employing advanced techniques. In the current study, we employed structural covariance (SC) model to investigate the development of triple networks in ASD. Three hundred and seven ASD and 337 typical controls were collected and further classified into four distinct age cohorts. Night brain seeds belonging to default modal network, salience network, and central executive network were obtained. SC between those seeds, as well as its topological properties, was calculated for each group within each age cohort. Statistical analysis revealed that ASD had dynamic reconfigurations of SC of the triple networks, especially the right fronto-insular cortex. The results might indicate that ASD had specific mechanism within distinct age cohort. Additionally, the big data sharing, together with the SC modal, was able to facilitate understanding of the mechanism underlying ASD.
\end{abstract}

Keywords: Big data, Structural covariance, Triple networks, Autism spectrum disorder

\section{Background}

In the last decades, much progress has been made to the data open sharing, especially in the neuroimaging studies of brain function and disease (Russell et al. 2014). On the one hand, the increasing big data sharing has profound impact on research in cognitive neuroscience and psychiatry, resulting in advances in the diagnosis and treatment of psychiatric and neurological disease. On the other hand, the big data sharing is able to facilitate development of advanced analysis methods, which in turn help find the new biomarkers in brain diseases. The structural covariance (SC) model is such a method that has its potential value in understanding of various psychiatric conditions (Aaron et al. 2013).

The SC is a phenomenon that inter-individual differences in the structure of a brain region often covary with the inter-individual structural difference of other brain areas. It has been recognized that the genetics, behavior, and plasticity together contributed to covariance between brain areas (Krista et al. 2009; William et al. 2001). The SC is usually characterized by the linear dependence between two large samples of human datasets using the product-moment correlation coefficient, the Pearson's $r$. The method has been widely employed to investigate the development of brain structures across lifespan (Brandon et al. 2010), and also the neurodegenerative disorders, such as the Alzheimer's

(c) The Author(s) 2016. This article is distributed under the terms of the Creative Commons Attribution 4.0 International License (http://creativecommons.org/licenses/by/4.0/), which permits unrestricted use, distribution, and reproduction in any medium, provided you give appropriate credit to the original author(s) and the source, provide a link to the Creative Commons license, and indicate if changes were made. 
disease (Yong et al. 2008) and Schizophrenia (Serge et al. 2005). Question surrounding the SC in autism spectrum disorder (ASD) still remains.

The ASD is a neurodevelopment disorder characterized by deficits in communication and social interaction, along with repetitive patterns of behavior and interests. Neuroimaging studies have demonstrated that dysfunction of triple networks [including central executive network (CEN), salience network (SN), and default modal network (DMN)] were associated with ASD (Vinod et al. 2011). To the best of our knowledge, there were no studies investigating the SC of those networks, especially their dynamic configuration across ages in ASD. The lack of investigation of the SC in ASD is probably due to the limited number of participants recruited in previous studies. Therefore, in the current study, we collected big datasets from the Autism Brain Imaging Data Exchange (ABIDE). Then we employed the SC modal to investigate the SC and topological properties of the triple networks and their development across ages in ASD.

\section{Methods}

\section{Participants and data preprocessing}

The datasets supporting the conclusions of this article are available from the ABIDE (http://fcon_1000.projects.nitrc.org/indi/abide/) database. The datasets involved 307 ASD and 337 typical controls (TC). Participants were further classified into four groups based on different age cohorts. They are group 1 (59 ASD, 63 TC, age, 6-11 years), group 2 (109 ASD, 114 TC, age, 11-15 years), group 3 (46 ASD, 52 TC, age, 15-18 years), and group 4 (93 ASD, 107 TC, age, >18 years). Written informed consent was obtained from all participants. Experimental protocols were approved by the local Institutional Review Boards.

All participants were scanned using a 3 Tesla SIEMENS scanner following diagnostic assessment. Subjects were asked to relax and look at a white cross-hair against a black background. The anatomical image was then acquired for each participant.

Data preprocessing was conducted using SPM8 software. First, all T1-weighted anatomical images were manually reoriented to place the anterior commissure at the origin of the three-dimensional Montreal Neurological Institute (MNI) space. The images were then segmented into gray matter, white matter, and cerebrospinal fluid (John et al. 2005). A diffeomorphic non-linear registration algorithm was used to spatially normalize the segmented images (John et al. 2007). This procedure generated a template for a group of individuals. The resulting images were spatially normalized into the MNI space using affine spatial normalization, and resampled into $1.5 \times 1.5 \times 1.5 \mathrm{~mm}^{3}$. Finally, the resulting gray matter images were smoothed with a $6 \mathrm{~mm}$ full-width half-maximum (FWHM) isotropic Gaussian kernel.

\section{Structural connectivity estimation}

Nine coordinates in MNI space were obtained from a previous study (Lucina et al. 2011). The brain areas corresponding to those coordinates were left and right fronto-insular cortex (IFIC/rFIC), anterior cingulate cortex (ACC), left and right dorsolateral prefrontal cortex (IDLPFC/rDLPFC), left and right posterior parietal cortex (IPPC/rPPC), ventromedial prefrontal cortex (VMPFC), and posterior cingulate cortex (PCC). The IFIC/ rFIC and ACC belong to SN. The IDLPFC/rDLPFC and IPPC/rPPC belong to CEN. The 
VMPFC and PCC belong to DMN. Night spherical regions of interests (ROIs) were generated with radius of $8 \mathrm{~mm}$ based on those coordinates. Those ROIs were multiplied with a gray matter mask to exclude voxels outsides the gray matter. The gray matter density value was averaged across voxels within each ROI for each participant. The SC analysis was then conducted as follows:

The Pearson correlation analysis was performed between pairs of ROIs to characterize the $\mathrm{SC}$ in ASD and HC. The permutation test was employed to determine the statistical significance level. Briefly, we first calculated the between-group difference of the correlation value. We then randomly assigned each participant to one of the two groups with the same size as the origin groups of ASD and HC. This randomization procedure was repeated for 10,000 permutations, which generated a null permutation distribution. For each permutation, the new between-group difference was calculated. We then assigned a $\mathrm{p}$ value to the between-group difference by computing the proportion of differences exceeding the null distribution values. The multiple comparisons were corrected using an exploratory threshold of $1 / \mathrm{N}$ (here, $\mathrm{N}$ is number of edges, which is $9 * 8 / 2$ ). Notably, the effect of sites and full IQ were regressed out before the Pearson correlation analysis. The whole procedure mentioned above was repeatedly conducted on each of the four groups.

\section{Topological properties calculation}

Within each age cohort, we computed the topological properties of SC networks including clustering coefficient, shortest path length, local efficiency and global efficiency, for ASD group and HC group, and compared those properties between the two groups.

The connectivity sparsity was firstly employed to threshold SC network. Connectivity sparsity was computed as number of existing edges divided by maximum possible number of edge in a network. Here, a connectivity sparsity of $40 \%$ was used to ensure that all SC networks were full connected. We then computed those topological properties for each SC network.

The clustering coefficient of a node $i$ is calculated as:

$$
C_{i}^{\mathrm{w}}=\frac{1}{k_{i}\left(k_{i}-1\right)} \sum_{j, h \in N}\left(w_{i j} w_{i h} w_{j h}\right)^{1 / 3},
$$

where $w_{i j}$ is the weight between node $i$ and node $j ; k_{i}$ is the degree of node $i$; $N$ is the number of nodes. The clustering coefficient of a network is computed as:

$$
C^{\mathrm{w}}=\frac{1}{N} \sum_{i \in N} C_{i}^{\mathrm{w}}
$$

This measure indicates the extent of local interconnectivity or cliquishness in network. The characteristic shortest path length of a network is defined as:

$$
L^{\mathrm{w}}=\frac{N(N-1)}{\sum_{i=1}^{N} \sum_{j \neq i}^{N} 1 / L_{i j}},
$$


where $L_{i j}$ is the path between node $i$ and node $j$ with shortest length. This measure quantifies the ability for information propagation in parallel.

The global efficiency of a network is computed as:

$$
E_{\text {global }}=\frac{1}{N(N-1)} \sum_{i \neq j \in N} \frac{1}{L_{i j}}
$$

which is a measure of parallel information transformation.

While the local efficiency of a network is defined as:

$$
E_{\mathrm{local}}=\frac{1}{N} \sum_{i \in G} E_{\text {global }}\left(G_{i}\right)
$$

where $E_{\text {global }}\left(G_{i}\right)$ is the global efficiency of the neighborhood sub-graph $G_{i}$ of the node $i$. The local efficiency can be understood as a measure of fault tolerance of the network, indicating how well each sub-graph exchanges information when the index node is eliminated.

The statistical test was conducted using permutation test, as we did in the "Participants and data preprocessing" section. The statistical level of $\mathrm{p}<0.05$ was considered as significant.

\section{Results}

The gray matte density value of each ROI strongly covaries with the values of rest ROIs in both ASD group and HC group (Fig. 1a). The SC value within SN was much higher, compared to those within DMN and CEN, and also those between the three networks (Fig. 1a). Further, ASD had significant reduced SC value between IPPC and VMPFC (Fig. 1b).
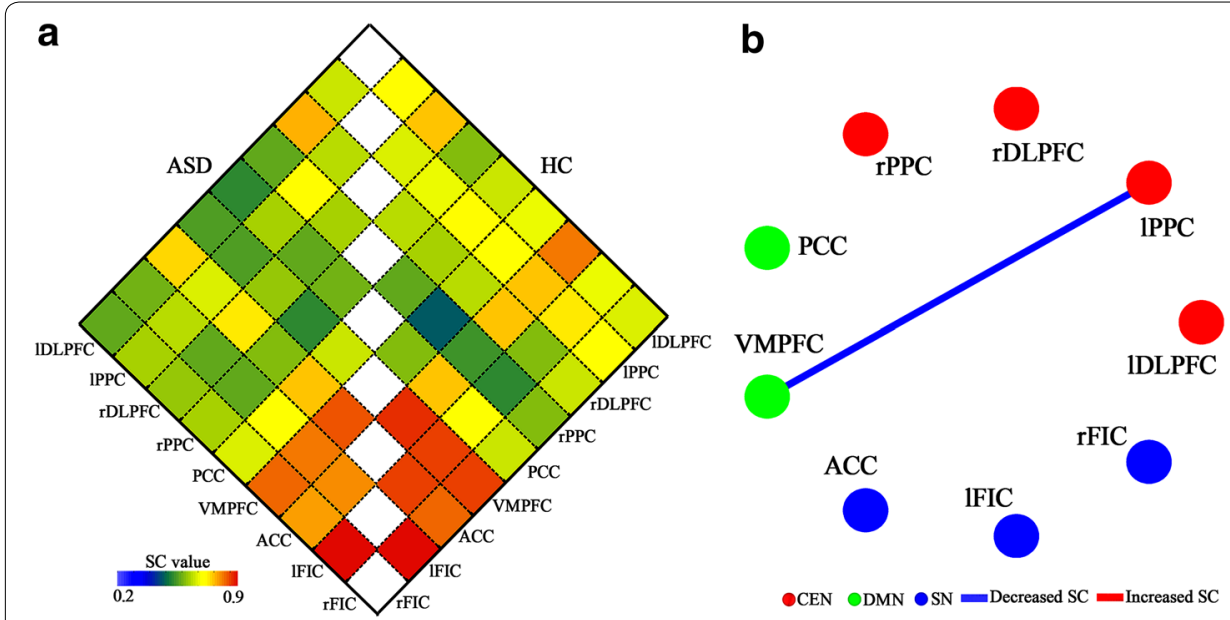

Fig. 1 The SC connectivity matrices across ages. The SC connectivity matrices in ASD and HC (a), and the statistical differences in SC value between ASD and $\mathrm{HC}(\mathbf{b})$. The value in the matrix represents the extent to which one brain area covary with other areas. Notably, these results were obtained by including all participants in ASD group and HC group 
We then observed different SC pattern between distinct age cohorts. In group 1, ASD had increased SC between ACC and IFIC, between ACC and VMPFC, and between rFIC and IFIC. In group 2, ASD had reduced SC between IPPC and rFIC, IFIC and VMPFC, between ACC and rFIC, IFIC. In group 3, increased SC was found in ASD between rPPC and rFIC. While in group 4, decreased SC was observed in ASD between ACC and IFIC, between VMPFC and IFIC (Fig. 2).

We further found that patients with ASD had significantly higher clustering coefficient at early adolescent, and lower global efficiency at late adolescent, compared to HC (Fig. 3). There was no difference in characteristic shortest path length and local efficiency between ASD and TC within each cohort.
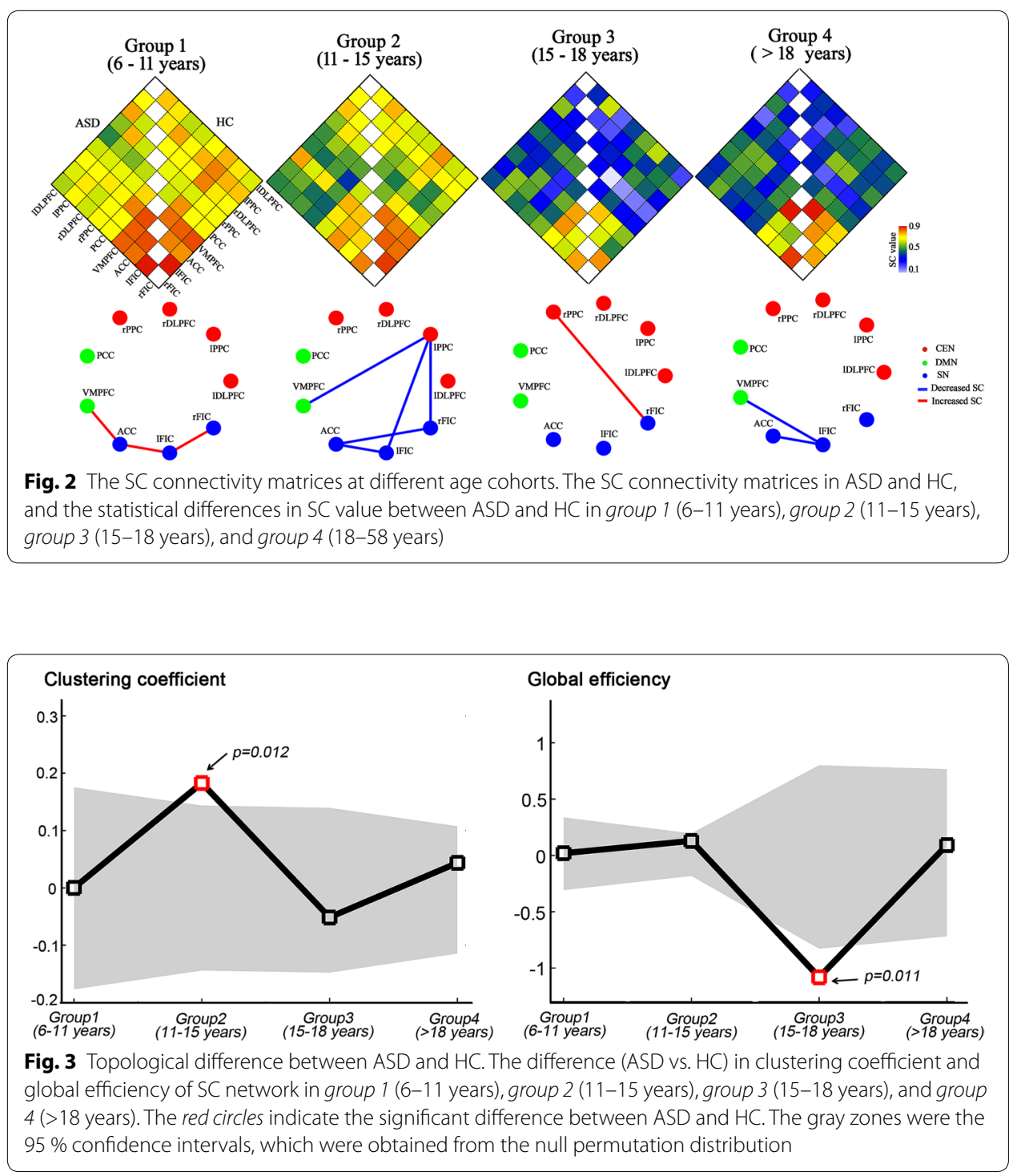


\section{Discussion}

In the current study, we observed dynamic SC reorganization of triple networks, especially the rFIC, by employing the SC model. It has been demonstrated that the rFIC, a critical component of SN, mediates interaction between CEN and DMN (Vinod et al. 2010; William et al. 2007). A developmental study had shown that the maturation of rFIC connectivity plays a critical role in the brain network maturation to support complex cognitive processes (Lucina et al. 2011). Those results probably suggested abnormal development of cognitive processes in ASD.

More specifically, we firstly found decreased SC between IPPC and VMPFC, which might suggest impaired executive function in ASD (Timothy et al. 2006). Interestingly, we then observed dramatically distinct SC patterns, especially the connectivity of rFIC in ASD across age cohorts. The findings here provided structural substrates for functional deficits of rFIC observed in ASD (Jyri-Johan et al. 2010), and might suggest specific mechanism underlying ASD in different age cohort. Additionally, a lower global efficiency in ASD was observed at specific age cohort, indicating that the altered information communication pattern within the triple networks was affected by ages. Overall, we were the first to report the dynamic SC reconfiguration of the triple networks in ASD using SC modal, and highlighted the importance of age effect in autistic research.

\section{Conclusions}

This study investigated the dynamic changes of SC and its topological properties as function of age cohorts in patents with ASD by employing a large dataset. The results suggested the crucial role of triple network abnormalities in pathology of ASD at specific age ranges, and highlighted effect of age on autistic development.

Authors' contributions

$\mathrm{HC}$ and $\mathrm{ZL}$ proposed and implemented the idea. ZL, HC, and YZ performed the data analysis. ZL and XD drafted the manuscript. All authors read and approved the final manuscript.

\section{Acknowledgements}

The work is supported by 863 project (2015AA020505) and the Natural Science Foundation of China (61533006 and 81301279).

Competing interests

The authors declare that they have no competing interests.

Received: 13 September 2016 Accepted: 8 October 2016

Published online: 21 October 2016

References

Aaron AB, Jay NG, Ed B (2013) Imaging structural co-variance between human brain regions. Nat Rev Neurosci $14: 322-336$

Brandon AZ, Efstathios DG, Juan Z, William WS (2010) Network-level structural covariance in the developing brain. Proc Natl Acad Sci 107:18191-18196

John A (2007) A fast diffeomorphic image registration algorithm. Neuroimage 38:95-113

John A, Karl JF (2005) Unified segmentation. Neuroimage 26:839-851

Jyri-Johan P, Jukka R, Xiangyu L, Irma M, Osmo T, Juha N, Tuemo S, Jukka R, Tuula H, Helena H, Katja J, Sanna K, Marja-

Leena M, Yufeng Z, Vesa K (2010) Alterations in regional homogeneity of resting-state brain activity in autism spectrum disorders. Brain Res 1321:169-179

Krista LH, Jason L, Andrea N, Marie F, Ellen W, Alan E, Gottfried S (2009) Musical training shapes structural brain development. J Neurosci 29:3019-3025

Lucina QU, Kaustubh SS, Srikanth R, Vinod M (2011) Dynamic reconfiguration of structural and functional connectivity across core neurocognitive brain networks with development. J Neurosci 31:18578-18589

Russell AP, Krzysztof JG (2014) Making big data open: data sharing in neuroimaging. Nat Neurosci 17:1510-1517 
Serge AM, Monte SB, Adam MB, Lina S (2005) Cortical intercorrelations of frontal area volumes in schizophrenia. Neuroimage 27:753-770

Timothy JS, Nicole R, John LB, Bruce T, Gary E, Michael WO, Ross C (2006) Visuospatial processing and the function of prefrontal-parietal networks in autism spectrum disorders: a functional MRI study. Am J Psychiatry 163:1440-1443

Vinod M (2011) Large-scale brain networks and psychopathology: a unifying triple network model. Trends Cogn Sci 15:483-506

Vinod M, Lucina QU (2010) Saliency, switching, attention and control: a network model of insula function. Brain Struct Funct 214:655-667

William FB, Hilleke EP, Dorret IB, Danielle P, Eco JG, Hugo GS, Neeltje EH, Clarine JO, Rene SK (2001) Quantitative genetic modeling of variation in human brain morphology. Cereb Cortex 11:816-824

William WS, Vinod M, Alan FS, Jennifer K, Gary HG, Heather K, Allan LR, Michael DG (2007) Dissociable intrinsic connectivity networks for salience processing and executive control. J Neurosci 27:2349-2356

Yong $\mathrm{H}$, Xhang C, Alan E (2008) Structural insights into aberrant topological patterns of large-scale cortical networks in Alzheimer's disease. J Neurosci 28:4756-4766

\section{Submit your manuscript to a SpringerOpen ${ }^{\circ}$ journal and benefit from:}

- Convenient online submission

\section{- Rigorous peer review}

- Immediate publication on acceptance

- Open access: articles freely available online

- High visibility within the field

- Retaining the copyright to your article

Submit your next manuscript at $\gg$ springeropen.com 